\title{
The Lieb-Yau Conjecture for Ground States of Pseudo-Relativistic Boson Stars
}

\author{
Yujin Guo* and Xiaoyu Zeng ${ }^{\dagger}$
}

April 16, 2019

\begin{abstract}
It is known that ground states of the pseudo-relativistic Boson stars exist if and only if the stellar mass $N>0$ satisfies $N<N^{*}$, where the finite constant $N^{*}$ is called the critical stellar mass. Lieb and Yau conjecture in [Comm. Math. Phys., 1987] that ground states of the pseudo-relativistic Boson stars are unique for each $N<N^{*}$. In this paper, we prove that the above uniqueness conjecture holds for the particular case where $N>0$ is small enough.
\end{abstract}

Keywords: Uniqueness; Ground states; Boson stars; Pohozaev identity

\section{Introduction}

Various models of pseudo-relativistic boson stars have attracted a lot of attention in theoretical and numerical astrophysics over the past few decades, see [29, 30] and references therein. In this paper, we are interested in ground states of pseudo-relativistic Boson stars in the mean field limit (cf. [10, 14, 29]), which can be described by constraint minimizers of the following variational problem

$$
e(N):=\inf \left\{\mathcal{E}(u): u \in H^{\frac{1}{2}}\left(\mathbb{R}^{3}\right) \text { and } \int_{\mathbb{R}^{3}}|u(x)|^{2} d x=N\right\},
$$

where $N>0$ denotes the stellar mass of Boson stars, and the pseudo-relativistic Hartree energy functional $\mathcal{E}(u)$ is of the form

$$
\mathcal{E}(u):=\int_{\mathbb{R}^{3}} \bar{u}\left(\sqrt{-\Delta+m^{2}}-m\right) u d x-\frac{1}{2} \int_{\mathbb{R}^{3}}\left(|x|^{-1} *|u|^{2}\right)|u|^{2} d x, \quad m>0 .
$$

Here the operator $\sqrt{-\Delta+m^{2}}$ is defined via multiplication in the Fourier space with the symbol $\sqrt{|\xi|^{2}+m^{2}}$ for $\xi \in \mathbb{R}^{3}$, which describes the kinetic and rest energy of many self-gravitating and relativistic bosons with rest mass $m>0$, and the symbol $*$ stands

*Email: yguo@mail.ccnu.edu.cn.

${ }^{\dagger}$ Email: xyzeng@whut.edu.cn. 
for the convolution on $\mathbb{R}^{3}$. Because of the physical relevance, without special notations we always focus on the case $m>0$ throughout the whole paper. The main purpose of this paper is to prove the uniqueness of minimizers for $e(N)$, provided that $N>0$ is small enough.

The variational problem $e(N)$ is essentially in the class of $L^{2}$-critical constraint minimization problems, which were studied recently in the nonrelativistic cases, e.g. [5, 19, 20, 22] and references therein. Comparing with these mentioned works, it however deserves to remark that the analysis of $e(N)$ is more complicated in a substantial way, which is mainly due to the nonlocal nature of the pseudo-differential operator $\sqrt{-\Delta+m^{2}}$, and the convolution-type nonlinearity as well. Starting from the pioneering papers [29, 30], many works were devoted to the mathematical analysis of the variational problem $e(N)$ over the past few years, see [10, 12, 13, 14, 15, 23, 26, 33, 37] and references therein. The existing results show that the analysis of $e(N)$ is connected well to the following Gagliardo-Nirenberg inequality of fractional type

$$
\int_{\mathbb{R}^{3}}\left(\frac{1}{|x|} *|u|^{2}\right)|u|^{2} d x \leq \frac{2}{\|w\|_{2}^{2}}\left\|(-\Delta)^{1 / 4} u\right\|_{2}^{2}\|u\|_{2}^{2}, \quad u \in H^{\frac{1}{2}}\left(\mathbb{R}^{3}\right)
$$

where $w(x)=w(|x|)>0$ is a ground state, up to translations and suitable rescaling (cf. [14, 29]), of the fractional equation

$$
\sqrt{-\Delta} u+u-\left(\frac{1}{|x|} *|u|^{2}\right) u=0 \text { in } \mathbb{R}^{3}, \text { where } u \in H^{\frac{1}{2}}\left(\mathbb{R}^{3}\right) .
$$

By making full use of (1.3), Lenzmann in [26] established the following interesting existence and analytical characters of minimizers for $e(N)$ :

Theorem A ([26, Theorem 1]) Under the assumption $m>0$, the following results hold for $e(N)$ :

1. $e(N)$ has minimizers if and only if $0<N<N^{*}$, where the finite constant $N^{*}$ is independent of $m$.

2. Any minimizer $u$ of $e(N)$ satisfies $u \in H^{s}\left(\mathbb{R}^{3}\right)$ for all $s \geq \frac{1}{2}$.

3. Any nonnegative minimizer of e $(N)$ must be strictly positive and radially symmetricdecreasing, up to phase and translation.

We remark that the existence of the critical constant $N^{*}>0$ stated in Theorem A, which is called the critical stellar mass of boson stars, was proved earlier in [14, 29]. Further, the dynamics and some other analytic properties of minimizers for $e(N)$ were also investigated by Lenzmann and his collaborators in [12, 13, 14, 15, 25, 26] and references therein. Stimulated by [20, 22], the related limit behavior of minimizers for $e(N)$ as $N \nearrow N^{*}$ were studied more recently in [23, 33, 37, where the Gagliardo-Nirenberg type inequality (1.3) also played an important role. Note also from the variational theory that any minimizer $u$ of $e(N)$ satisfies the Euler-Lagrange equation

$$
\left(\sqrt{-\Delta+m^{2}}-m\right) u-\left(\frac{1}{|x|} *|u|^{2}\right) u=-\mu u \text { in } \mathbb{R}^{3},
$$


where $\mu \in \mathbb{R}$ is the associated Lagrange multiplier. Following (1.5) and Theorem A, one can deduce that any minimizer of $e(N)$ must be either positive or negative, see [23] for details. Therefore, it is enough to consider positive minimizers of $e(N)$, which are called ground states of $e(N)$ throughout the rest part of this paper.

Whether a physics system admits a unique ground state or not is an interesting and fundamental problem. Lieb and Yau [29] conjectured in 1987 that for each $N<N^{*}$, there exists a unique ground state (minimizer) of $e(N)$. As expected by Lieb and Yau there, the analysis of this uniqueness conjecture is however challenging extremely. Actually, it is generally difficult to prove whether any two different ground states of $e(N)$ satisfy the equation (1.5) with the same Lagrange multiplier $\mu \in \mathbb{R}$. On the other hand, more difficultly, it seems very challenging to address the uniqueness of ground states for (1.5). Essentially, even though the uniqueness of ground states for the following fractional equation

$$
(-\Delta)^{s} u+u-u^{\alpha+1}=0 \text { in } \mathbb{R}^{N}, u \in H^{s}\left(\mathbb{R}^{N}\right),
$$

where $0<s<1, \alpha>0$ and $N \geq 1$, was already proved in the celebrated works [11, 12, the uniqueness of ground states for (1.4) or (1.5) is still open, due to the nonlocal nonlinearity of Hartree type. Therefore, whether the above Lieb-Yau conjecture is true for all $0<N<N^{*}$ remains mainly open after three decades, except Lenzmann's recent work [26] in 2009.

As an important first step towards the Lieb-Yau conjecture, Lenzmann proved in [26] that for each $0<N \ll N^{*}$ and except for at most countably many $N$, the uniqueness of minimizers for $e(N)$ holds true. We emphasize that the additional assumption "except for at most countably many $N$ " seems essential in Lenzmann's proof, since the smoothness of the GP energy $e(N)$ with respect to $N$ was employed there. In this paper we intend to remove the above additional assumption and prove the Lieb-Yau conjecture in the particular case where $0<N<N^{*}$ is small enough. More precisely, our main result of this paper is the following uniqueness of minimizers for $e(N)$.

Theorem 1.1. If $N>0$ is small enough, then the problem e $(N)$ admits a unique positive minimizer, up to phase and translation.

The similar uniqueness results of Theorem 1.1 were established recently in [2, Theorem 2] and [31, Theorem 1.1] (see also [21, Corollary 1.1]) for the nonrelativistic Hartree minimization problems with trapping potentials, under the additional assumption that the associated nonrelativistic operator admits the first eigenvalue. We however emphasize that the arguments of [2, 31, 21] are not applicable for proving Theorem 1.1, since the associated pseudo-relativistic operator $H:=\sqrt{-\Delta+m^{2}}-m$ does not admit any eigenvalue in our problem $e(N)$. Therefore, a different approach is needed for proving Theorem 1.1. Towards this purpose, since positive minimizers $u$ of $e(N)$ vanish uniformly as $N \searrow 0$, motivated by [26] we define

$$
\tilde{u}(x)=c^{2} u(c x) \text {, where } c>0,
$$

so that

$$
\mathcal{E}(u)=c^{-3} \mathcal{E}_{c}(\tilde{u}) \text { and } \int_{\mathbb{R}^{3}}|\tilde{u}|^{2} d x=c \int_{\mathbb{R}^{3}}|u|^{2} d x
$$


where the energy functional $\mathcal{E}_{c}(\cdot)$ is given by

$$
\mathcal{E}_{c}(u):=\int_{\mathbb{R}^{3}} \bar{u}\left(\sqrt{-c^{2} \Delta+c^{4} m^{2}}-c^{2} m\right) u d x-\frac{1}{2} \int_{\mathbb{R}^{3}}\left(|x|^{-1} *|u|^{2}\right)|u|^{2} d x, \quad m>0 .
$$

Consider the minimization problem

$$
\bar{e}(c):=\inf \left\{\mathcal{E}_{c}(u): u \in H^{\frac{1}{2}}\left(\mathbb{R}^{3}\right) \text { and } \int_{\mathbb{R}^{3}}|u(x)|^{2} d x=1\right\} .
$$

Note from Theorem A and (1.7) that if $c>0$ is large enough, then $\bar{e}(c)$ in (1.9) admits positive minimizers. More importantly, setting $c=N^{-1}>0$, studying positive minimizers of $e(N)$ as $N \searrow 0$ is then equivalent to investigating positive minimizers of the minimization problem (1.9) as $c \nearrow \infty$. Therefore, to establish the uniqueness of Theorem 1.1, it suffices to prove the following uniqueness theorem.

Theorem 1.2. If $c>0$ is large enough, then $\bar{e}(c)$ in (1.9) admits a unique positive minimizer, up to phase and translation.

Suppose now that $Q_{c}>0$ is a minimizer of $\bar{e}(c)$ defined in (1.9). Then there exists a Lagrange multiplier $\mu_{c} \in \mathbb{R}$ such that $Q_{c}>0$ solves

$$
\left(\sqrt{-c^{2} \Delta+c^{4} m^{2}}-c^{2} m\right) Q_{c}-\left(\frac{1}{|x|} *\left|Q_{c}\right|^{2}\right) Q_{c}=-\mu_{c} Q_{c} \text { in } \mathbb{R}^{3} .
$$

Recall from [26, Proposition 1] that up to a subsequence if necessary, $\mu_{c} \in \mathbb{R}$ satisfies

$$
\mu_{c} \rightarrow-\lambda<0 \text { as } c \rightarrow \infty
$$

for some constant $\lambda>0$. In order to prove Theorem 1.2, associated to $Q_{c}$, we need to study the uniformly exponential decay of $\phi_{c} \in H^{\frac{1}{2}}\left(\mathbb{R}^{3}\right)$ as $c \rightarrow \infty$, where $\phi_{c}$ satisfies

$$
\begin{aligned}
& \left(\sqrt{-c^{2} \Delta+m^{2} c^{4}}-m c^{2}\right) \phi_{c}-\left(\frac{1}{|x|} * Q_{c}^{2}\right) \phi_{c}-2 k_{1}\left\{\frac{1}{|x|} *\left(Q_{c} \phi_{c}\right)\right\} Q_{c} \\
& -k_{2}(c) Q_{c}=-\mu_{c} \phi_{c} \text { in } \mathbb{R}^{3} .
\end{aligned}
$$

Here $\mu_{c} \in \mathbb{R}$ satisfies (1.11), $k_{1} \geq 0$ and $k_{2}(c) \in \mathbb{R}$ is bounded uniformly in $c>0$. As proved in Lemma 2.1, we shall derive the following uniformly exponential decay of $\phi_{c}$ as $c \rightarrow \infty$ :

$$
\left|\phi_{c}(x)\right| \leq C e^{-\delta|x|} \text { in } \mathbb{R}^{3}
$$

holds uniformly as $c \rightarrow \infty$, where the constants $\delta>0$ and $C=C(\delta)>0$ are independent of $c$. Since $\mu_{c} \in \mathbb{R}$ satisfies (1.11), stimulated by [1, 14, 24, 35], the proof of (1.13) is based on the uniformly exponential decay (2.25) of the Green's function $G_{c}(\cdot)$ for $\left(\bar{H}_{c}+\mu_{c}\right)^{-1}$ as $c \rightarrow \infty$, where the operator $\bar{H}_{c}$ is defined by

$$
\bar{H}_{c}:=\sqrt{-c^{2} \Delta+m^{2} c^{4}}-m c^{2} .
$$

As shown in Lemma 2.3, it however deserves to remark that because $G_{c}(\cdot)$ depends on $c>0$, one needs to carry out more delicate analysis, together with some tricks, 
for addressing the uniformly exponential decay (2.25) of $G_{c}(\cdot)$ as $c \rightarrow \infty$. On the other hand, as a byproduct, the exponential decay (1.13) can be useful in analyzing the limiting procedure of solutions for Schrodinger equations involving the above fractional operator $\bar{H}_{c}$, which were investigated widely in [6, 7, 8] and references therein.

Following (1.13) and the regularity of $Q_{c}$, in Section 2 we shall finally prove the following limit behavior

$$
Q_{c} \rightarrow Q_{\infty} \text { uniformly in } \mathbb{R}^{3} \text { as } c \rightarrow \infty,
$$

where $Q_{\infty}>0$ is the unique positive minimizer of (2.1) described below. Based on the refined estimates of Section 2, motivated by [9, 18, 19], we shall employ the nondegenerancy and uniqueness of $Q_{\infty}$ to complete the proof of Theorem 1.2 by establishing Pohozaev identities.

This paper is organized as follows. In Section 2 we shall address some refined estimates of $Q_{c}$ as $c \rightarrow \infty$, where Lemma 2.1 is proved in Subsection 2.1. Following those estimates of Section 2, Section 3 is devoted to the proof of Theorems 1.2 on the uniqueness of minimizers for $\bar{e}(c)$ as $c \rightarrow \infty$. Theorem 1.1 then follows immediately from Theorem 1.2 in view of the relation (1.7).

\section{Analytical Properties of $Q_{c}$ as $c \rightarrow \infty$}

The main purpose of this section is to give some refined analytical estimates of $Q_{c}$ as $c \rightarrow \infty$, where $Q_{c}>0$ is a positive minimizer of $\bar{e}(c)$ defined in (1.9). Note also from Theorem A and (1.7) that $Q_{c}$ is radially symmetric in $|x|$.

We first introduce the following limit problem associated to $\bar{e}(c)$ :

$$
e_{m}:=\inf \left\{E_{m}(u): u \in H^{1}\left(\mathbb{R}^{3}\right) \text { and } \int_{\mathbb{R}^{3}}|u(x)|^{2} d x=1\right\}
$$

where the energy functional $E_{m}(\cdot)$ satisfies

$$
E_{m}(u):=\frac{1}{2 m} \int_{\mathbb{R}^{3}}|\nabla u|^{2} d x-\frac{1}{2} \int_{\mathbb{R}^{3}}\left(|x|^{-1} *|u|^{2}\right)|u|^{2} d x, \forall u \in H^{1}\left(\mathbb{R}^{3}\right), \quad m>0 .
$$

For any given $m>0$, it is well-known that, up to translations, problem (2.1) has a unique positive minimizer denoted by $Q_{\infty}(|x|)$, which must be radially symmetric, see [26, 27] and references therein. Further, $Q_{\infty}>0$ solves the following equation

$$
-\frac{1}{2 m} \Delta Q_{\infty}+\lambda Q_{\infty}=\left(|x|^{-1} *\left|Q_{\infty}\right|^{2}\right) Q_{\infty} \text { in } \mathbb{R}^{3}, \quad Q_{\infty} \in H^{1}\left(\mathbb{R}^{3}\right),
$$

where the Lagrange multiplier $\lambda>0$ depends only on $m$ and is determined uniquely by the constraint condition $\int_{\mathbb{R}^{3}} Q_{\infty}^{2} d x=1$. Note from [32, Theorem 3] that $Q_{\infty}>0$ is a unique positive solution of (2.3). Moreover, recall from [26, Theorem 4] that $Q_{\infty}$ is non-degenerate, in the sense that the linearized operator $L_{+}: H^{2}\left(\mathbb{R}^{3}\right) \mapsto L^{2}\left(\mathbb{R}^{3}\right)$, which is defined by

$$
L_{+} \xi:=\left(-\frac{1}{2 m} \Delta+\lambda-|x|^{-1} *\left|Q_{\infty}\right|^{2}\right) \xi-2\left(|x|^{-1} *\left(Q_{\infty} \xi\right)\right) Q_{\infty},
$$


satisfies

$$
\operatorname{ker} L_{+}=\operatorname{span}\left\{\frac{\partial Q_{\infty}}{\partial x_{1}}, \frac{\partial Q_{\infty}}{\partial x_{2}}, \frac{\partial Q_{\infty}}{\partial x_{3}}\right\}
$$

As a positive minimizer of $\bar{e}(c), Q_{c}>0$ satisfies the following equation

$$
\left(\sqrt{-c^{2} \Delta+m^{2} c^{4}}-m c^{2}\right) Q_{c}-\left(|x|^{-1} * Q_{c}^{2}\right) Q_{c}=\mu_{c} Q_{c} \text { in } \mathbb{R}^{3},
$$

where $\mu_{c} \in \mathbb{R}$ is a suitable Lagrange multiplier. Recall from [26, Proposition 1] that up to a subsequence if necessary, the Lagrange multiplier $\mu_{c}$ of (2.6) satisfies

$$
\mu_{c} \rightarrow-\lambda<0 \text { as } c \rightarrow \infty
$$

where $\lambda>0$ is the same as that of (2.3). Associated to the positive minimizer $Q_{c}>0$ of (2.6), we next define the linearized operator

$$
\begin{aligned}
\mathcal{L}_{k_{1}, k_{2}} \xi:= & \left(\sqrt{-c^{2} \Delta+m^{2} c^{4}}-m c^{2}\right) \xi-\left(\frac{1}{|x|} * Q_{c}^{2}\right) \xi \\
& -2 k_{1}\left\{\frac{1}{|x|} *\left(Q_{c} \xi\right)\right\} Q_{c}-k_{2}(c) Q_{c} \text { in } \mathbb{R}^{3},
\end{aligned}
$$

where the constants $m>0$ and $k_{1} \geq 0$, and $k_{2}(c) \in \mathbb{R}$ is bounded uniformly in $c>0$.

Lemma 2.1. Suppose $\varphi_{c}=\varphi_{c}(x) \in H^{\frac{1}{2}}\left(\mathbb{R}^{3}\right)$ is a solution of

$$
\mathcal{L}_{k_{1}, k_{2}} \varphi_{c}=\mu_{c} \varphi_{c} \text { in } \mathbb{R}^{3},
$$

where $\mu_{c}$ satisfies (2.7), and the operator $\mathcal{L}_{k_{1}, k_{2}}$ is defined by (2.8) for some constants $m>0$ and $k_{1} \geq 0$, and $k_{2}(c) \in \mathbb{R}$ being bounded uniformly in $c>0$. Then there exist $\delta>0$ and $C=C(\delta)>0$, which are independent of $c>0$, such that

$$
\left|\varphi_{c}(x)\right| \leq C e^{-\delta|x|} \text { in } \mathbb{R}^{3}
$$

uniformly for all sufficiently large $c>0$.

Since the proof of Lemma 2.1 is a little involved, we leave it to Subsection 2.1. Applying Lemma 2.1, we next address the following estimates of $Q_{c}>0$ as $c \rightarrow \infty$, which are crucial for the proof of Theorem 1.2 .

Proposition 2.2. Let $Q_{c}>0$ be a positive minimizer of $\bar{e}(c)$ defined in (1.9) as $c \rightarrow \infty$. Then we have

1. There exist $\delta>0$ and $C=C(\delta)>0$, which are independent of $c>0$, such that

$$
\left|Q_{c}(x)\right|,\left|\nabla Q_{c}(x)\right| \leq C e^{-\delta|x|} \text { in } \mathbb{R}^{3}
$$

uniformly as $c \rightarrow \infty$.

2. $Q_{c}$ satisfies

$$
Q_{c} \rightarrow Q_{\infty} \text { uniformly in } \mathbb{R}^{3} \text { as } c \rightarrow \infty,
$$

where $Q_{\infty}>0$ is the unique positive minimizer of (2.1). 
Proof. 1. Since $Q_{c}>0$ solves (2.6), the uniformly exponential decay (2.11) for $Q_{c}$ as $c \rightarrow \infty$ follows directly from (2.45) below. Because $\frac{\partial Q_{c}}{\partial x_{i}}$ satisfies (2.9) for $k_{1}=1$ and $k_{2}(c) \equiv 0$, where $i=1,2,3$, the exponential decay (2.11) holds for $\nabla Q_{c}$ by applying Lemma 2.1 .

2. Following [31, Lemma 4.9] and references therein, we first recall from [26, Proposition 1] that $Q_{c}$ satisfies

$$
Q_{c} \rightarrow Q_{\infty} \text { in } H^{1}\left(\mathbb{R}^{3}\right) \text { as } c \rightarrow \infty
$$

where the convergence holds for the whole sequence of $\{c\}$, due to the uniqueness of $Q_{\infty}>0$. Rewrite (2.6) as

$$
\begin{aligned}
\left(-\frac{1}{2 m} \Delta-\mu_{c}\right) Q_{c} & =\left(|x|^{-1} * Q_{c}^{2}\right) Q_{c}-\left(\sqrt{-c^{2} \Delta+m^{2} c^{4}}-m c^{2}+\frac{1}{2 m} \Delta\right) Q_{c} \\
& :=\left(|x|^{-1} * Q_{c}^{2}\right) Q_{c}-F_{c}(\nabla) Q_{c} \text { in } \mathbb{R}^{3},
\end{aligned}
$$

where we denote the pseudo-differential operator

$$
F_{c}(\nabla):=\sqrt{-c^{2} \Delta+m^{2} c^{4}}-m c^{2}-\frac{-\Delta}{2 m}
$$

with the symbol

$$
F_{c}(\xi):=\sqrt{c^{2}|\xi|^{2}+m^{2} c^{4}}-m c^{2}-\frac{|\xi|^{2}}{2 m}, \quad \xi \in \mathbb{R}^{3} .
$$

Recall from (2.11) that $Q_{c}$ decays exponentially as $|x| \rightarrow \infty$ for all sufficiently large $c>0$. Moreover, since the operator $\bar{H}_{c}:=\sqrt{-c^{2} \Delta+m^{2} c^{4}}-m c^{2}$ is uniformly bounded from below for all $c>0$, the similar argument of [14, Theorem 4.1(i)] or [7, Proposition 4.2 ] applied to (2.6) and (2.7) yields that

$$
Q_{c} \in H^{s}\left(\mathbb{R}^{3}\right) \text { for all } s \geq \frac{1}{2},
$$

which further implies the uniform smoothness of $Q_{c}$ in $c>0$, and

$$
Q_{c} \in L^{\infty}\left(\mathbb{R}^{3}\right) \text { uniformly in } c>0 \text {. }
$$

Applying the Taylor expansion, we obtain from (2.15) that for $|\xi| \geq \frac{m c}{2}$,

$$
\left|F_{c}(\xi)\right|=|c| \xi\left|\sqrt{1+\left(\frac{m c}{|\xi|}\right)^{2}}-m c^{2}-\frac{|\xi|^{2}}{2 m}\right| \leq \sqrt{5} c|\xi|+m c^{2}+\frac{|\xi|^{2}}{2 m} \leq \frac{36|\xi|^{4}}{m^{3} c^{2}}, \quad \xi \in \mathbb{R}^{3},
$$

and for $|\xi| \leq \frac{m c}{2}$,

$$
\left|F_{c}(\xi)\right|=\left|m c^{2} \sqrt{1+\left(\frac{|\xi|}{m c}\right)^{2}}-m c^{2}-\frac{|\xi|^{2}}{2 m}\right| \leq \frac{|\xi|^{4}}{8 m^{3} c^{2}}, \quad \xi \in \mathbb{R}^{3},
$$

due to the fact that $\left|\sqrt{1+t}-1-\frac{1}{2} t\right| \leq \frac{1}{8} t^{2}$ holds for all $0<t \leq \frac{1}{2}$. Following above estimates, we then derive from (2.15) and (2.16) that for sufficiently large $c>0$,

$$
\left\|F_{c}(\nabla) Q_{c}\right\|_{H^{s}\left(\mathbb{R}^{3}\right)} \leq \frac{M_{1}}{m^{3} c^{2}}\left\|Q_{c}\right\|_{H^{s+4}\left(\mathbb{R}^{3}\right)}<\frac{M_{2}}{m^{3} c^{2}} \text { for all } s \geq \frac{1}{2},
$$


where $M_{1}>0$ and $M_{2}>0$ are independent of $c>0$. Also, since

$$
\left\||x|^{-1} * Q_{c}^{2}\right\|_{L^{\infty}\left(\mathbb{R}^{3}\right)} \leq C\left(\left\|Q_{c}\right\|_{L^{4}\left(\mathbb{R}^{3}\right)}^{2}+\left\|Q_{c}\right\|_{L^{2}\left(\mathbb{R}^{3}\right)}^{2}\right),
$$

we derive from (2.16) that for any $p \geq 2$,

$$
\left\|\left(|x|^{-1} * Q_{c}^{2}\right) Q_{c}\right\|_{L^{p}\left(\mathbb{R}^{3}\right)} \leq C\left(\left\|Q_{c}\right\|_{L^{4}\left(\mathbb{R}^{3}\right)}^{2}+\left\|Q_{c}\right\|_{L^{2}\left(\mathbb{R}^{3}\right)}^{2}\right)\left\|Q_{c}\right\|_{L^{p}\left(\mathbb{R}^{3}\right)} \leq K_{p},
$$

where $K_{p}>0$ is independent of $c>0$. Employing (2.17) and (2.19), together with Sobolev imbedding theorem, the bootstrap argument applied to (2.14) yields that

$$
Q_{c} \rightarrow Q_{\infty} \text { uniformly on any compact domain of } \mathbb{R}^{3} \text { as } c \rightarrow \infty \text {. }
$$

On the other hand, one can easily deduce from (2.3) that $Q_{\infty}$ decays exponentially as $|x| \rightarrow \infty$. Together with (2.11), this indicates that for any $\varepsilon>0$, there exists a constant $R_{\varepsilon}>0$, independent of $c>0$, such that

$$
\left|Q_{c}(x)\right|,\left|Q_{\infty}(x)\right|<\frac{\varepsilon}{4} \text { for any }|x|>R_{\varepsilon},
$$

and hence,

$$
\sup _{|x|>R_{\varepsilon}}\left|Q_{c}(x)-Q_{\infty}(x)\right| \leq \sup _{|x|>R_{\varepsilon}}\left(\left|Q_{c}(x)\right|+\left|Q_{\infty}(x)\right|\right) \leq \frac{\varepsilon}{2} .
$$

Moreover, it follows from (2.20) that for sufficiently large $c>0$,

$$
\sup _{|x| \leq R_{\varepsilon}}\left|Q_{c}(x)-Q_{\infty}(x)\right| \leq \frac{\varepsilon}{2} .
$$

The above two estimates thus yield that for sufficiently large $c>0$,

$$
\sup _{x \in \mathbb{R}^{3}}\left|Q_{c}(x)-Q_{\infty}(x)\right| \leq \varepsilon,
$$

which implies that (2.12) holds true. The lemma is therefore proved.

\subsection{Uniformly exponential decay as $c \rightarrow \infty$}

In this Subsection, we address the proof of Lemma 2.1 on the uniformly exponential decay as $c \rightarrow \infty$. We remark that even though the proof of Lemma 2.1 is stimulated from [1, 14, 24, 35], as shown in proving Lemma 2.3 below, we need to carry out more delicate analysis together with some tricks.

We first suppose that $\varphi_{c} \in H^{\frac{1}{2}}\left(\mathbb{R}^{3}\right)$ is a solution of

$$
\left(\sqrt{-c^{2} \Delta+m^{2} c^{4}}-m c^{2}\right) \varphi_{c}-\left(\frac{1}{|x|} * \varphi_{c}^{2}\right) \varphi_{c}=-\lambda_{c} \varphi_{c} \text { in } \mathbb{R}^{3}
$$

where the constant $m>0$ and

$$
\lambda_{c} \rightarrow 2 \lambda>0 \text { as } c \rightarrow \infty
$$


for some positive constant $\lambda$. Define

$$
\bar{H}_{c}:=\sqrt{-c^{2} \Delta+m^{2} c^{4}}-m c^{2}, \quad V(x):=-\frac{1}{|x|} * \varphi_{c}^{2} .
$$

Therefore, $\varphi_{c}$ can be thought of as an eigenfunction of the Schrodinger operator $H:=$ $\bar{H}_{c}+V(x)$. Moreover, the argument of [14, Theorem 4.1] or [7, Proposition 4.2] gives that $\varphi_{c} \in H^{s}\left(\mathbb{R}^{3}\right)$ for all $s \geq \frac{1}{2}$, which implies the smoothness of $\varphi_{c}$. Further, the spectrum of $\bar{H}_{c}$ satisfies

$$
\sigma\left(\bar{H}_{c}\right)=\sigma_{e s s}\left(\bar{H}_{c}\right)=[0, \infty)
$$

for all $c>0$. Under the assumption (2.22), then $\left(\bar{H}_{c}+\lambda_{c}\right)^{-1}$ exists for all sufficiently large $c>0$, and (2.21) can be rewritten as

$$
\varphi_{c}(x)=-\left(\bar{H}_{c}+\lambda_{c}\right)^{-1} V(x) \varphi_{c}(x) .
$$

Note also from (2.21) and (2.23) that

$$
\varphi_{c}(x)=-\int_{\mathbb{R}^{3}} G_{c}(x-y) V(y) \varphi_{c}(y) d y
$$

where $G_{c}(x-y)$ is the Green's function of $\left(\bar{H}_{c}+\lambda_{c}\right)^{-1}$ defined in (2.23). The following lemma gives the uniformly exponential decay of $G_{c}(\cdot)$ as $c \rightarrow \infty$.

Lemma 2.3. Suppose $\lambda_{c} \in \mathbb{R}$ satisfies (2.22) for some $\lambda>0$. Then for each $0<\delta<$ $\min \left\{\frac{m}{2}, \sqrt{\lambda m}\right\}$, there exists a constant $M:=M(\delta)>0$, independent of $c>0$, such that the Green's function $G_{c}(x-y)$ of $\left(\bar{H}_{c}+\lambda_{c}\right)^{-1}$ satisfies

$$
\left|G_{c}(x-y)\right| \leq M \frac{e^{-\delta|x-y|}}{|x-y|^{2}} \text { in } \mathbb{R}^{3}
$$

uniformly for all sufficiently large $c>0$.

Proof. Under the assumption (2.22) , since $G_{c}(\cdot)$ is the Green's function of $\left(\bar{H}_{c}+\lambda_{c}\right)^{-1}$ defined in (2.23) for all sufficiently large $c>0$, we have

$$
G_{c}(z)=f_{c}^{-1}(z), \quad f_{c}(\mu)=\frac{1}{\sqrt{c^{2}|\mu|^{2}+m^{2} c^{4}}-m c^{2}+\lambda_{c}} \quad \text { for } \mu \in \mathbb{R}^{3},
$$

and $f_{c}^{-1}: \mathcal{S}^{\prime} \rightarrow \mathcal{S}^{\prime}$ denotes the inverse Fourier transform of $f_{c}$. We obtain from (2.26) that for all sufficiently large $c>0$,

$$
G_{c}(z)=f_{c}^{-1}(z)=(2 \pi)^{-3 / 2} \int_{\mathbb{R}^{3}} \frac{e^{i \mu \cdot z}}{\sqrt{c^{2}|\mu|^{2}+m^{2} c^{4}}-m c^{2}+\lambda_{c}} d \mu=\frac{1}{c} g_{c}^{-1}(z),
$$

where

$$
g_{c}(\mu)=\frac{1}{\sqrt{|\mu|^{2}+m^{2} c^{2}}-m c+\frac{\lambda_{c}}{c}}, \quad \mu \in \mathbb{R}^{3} .
$$


In view of (2.27), we next define

$$
H_{c}+\frac{\lambda_{c}}{c}:=\sqrt{-\Delta+m^{2} c^{2}}-m c+\frac{\lambda_{c}}{c},
$$

so that

$$
\left(H_{c}+\frac{\lambda_{c}}{c}\right)^{-1}=\int_{0}^{\infty} e^{-t \frac{\lambda_{c}}{c}} e^{-t H_{c}} d t=\int_{0}^{\infty} e^{-t\left(\frac{\lambda_{c}}{c}-m c\right)} e^{-t \sqrt{p^{2}+m^{2} c^{2}}} d t, \quad p=-i \nabla .
$$

Note from pp. 183 of [28] that

$$
e^{-t \sqrt{p^{2}+m^{2} c^{2}}}(z)=\frac{m^{2} c^{2}}{2 \pi^{2}} \frac{t}{t^{2}+|z|^{2}} K_{2}\left(m c \sqrt{t^{2}+|z|^{2}}\right), \quad z \in \mathbb{R}^{3}
$$

where $K_{2}(\cdot)>0$ denotes the modified Bessel function of the third kind. We then derive from above that

$$
G_{c}(z)=\frac{1}{c} g_{c}^{-1}(z)=\frac{m^{2} c}{2 \pi^{2}} \int_{0}^{\infty} e^{-t\left(\frac{\lambda_{c}}{c}-m c\right)} \frac{t}{t^{2}+|z|^{2}} K_{2}\left(m c \sqrt{t^{2}+|z|^{2}}\right) d t .
$$

Recall from [24] that there exist positive constants $\bar{M}_{1}$ and $\bar{M}_{2}$, independent of $c>0$, such that

$$
K_{2}(c m w) \leq \begin{cases}\frac{\bar{M}_{1}}{c^{2} m^{2} w^{2}} & \text { if } w<\frac{2}{m c}, \\ \frac{\bar{M}_{2} e^{-c m w}}{\sqrt{c m w}} & \text { if } \quad w \geq \frac{1}{m c},\end{cases}
$$

where $w>0$ is a real number. We next follow (2.29) and (2.30) to complete the proof by discussing separately the following two cases, which involve very complicated estimates together with some tricks:

(1). Case of $|z| \geq \frac{1}{m c}$. In this case, we have $\sqrt{t^{2}+|z|^{2}} \geq \frac{1}{m c}$ for all $t \geq 0$. We then obtain from (2.22), (2.29) and (2.30) that for all sufficiently large $c>0$,

$$
\begin{aligned}
\frac{2 \pi^{2}}{\bar{M}_{2} m^{\frac{3}{2}}} G_{c}(z) \leq & \sqrt{c} \int_{0}^{\infty} \frac{t}{\left(t^{2}+|z|^{2}\right)^{\frac{5}{4}}} e^{-t\left(\frac{\lambda}{c}-m c\right)-m c \sqrt{t^{2}+|z|^{2}}} d t \\
= & \int_{2 \sqrt{\frac{m}{\lambda}} c^{\frac{3}{2}}|z|}^{\infty} A(t, z) d t+\int_{c|z|}^{2 \sqrt{\frac{m}{\lambda}} c^{\frac{3}{2}}|z|} A(t, z) d t \\
& +\int_{\frac{1}{4} \sqrt{\frac{m}{\lambda}} c^{\frac{1}{2}}|z|}^{c|z|} A(t, z) d t+\int_{0}^{\frac{1}{4} \sqrt{\frac{m}{\lambda}} c^{\frac{1}{2}}|z|} A(t, z) d t \\
:= & I_{A}(z)+I_{B}(z)+I_{C}(z)+I_{D}(z),
\end{aligned}
$$

where $A(t, z)$ satisfies

$$
A(t, z):=\frac{t \sqrt{c}}{\left(t^{2}+|z|^{2}\right)^{\frac{5}{4}}} e^{-t\left(\frac{\lambda}{c}-m c\right)-m c \sqrt{t^{2}+|z|^{2}}} .
$$


For $I_{A}(z)+I_{D}(z)$, we note that if $t \geq 0$ satisfies

$$
t \geq 2 \sqrt{\frac{m}{\lambda}} c^{\frac{3}{2}}|z| \text { or } 0 \leq t \leq \frac{1}{4} \sqrt{\frac{m}{\lambda}} c^{\frac{1}{2}}|z|,
$$

then one can check that

$$
\sqrt{t^{2}+|z|^{2}} \geq \sqrt{1-\frac{\lambda}{m c^{2}}} t+\sqrt{\frac{\lambda}{m c}}|z| \geq\left(1-\frac{2 \lambda}{3 m c^{2}}\right) t+\sqrt{\frac{\lambda}{m c}}|z|
$$

for sufficiently large $c>0$. We thus obtain from (2.32) and (2.33) that for sufficiently large $c>0$,

$$
\begin{aligned}
I_{A}(z)+I_{D}(z) & \leq \int_{0}^{\infty} \frac{t \sqrt{c}}{\left(t^{2}+|z|^{2}\right)^{\frac{5}{4}}} e^{-\frac{\lambda}{3 c} t-\sqrt{\lambda m c}|z|} d t \\
& \leq C_{1} \frac{e^{-\sigma|z|}}{\sqrt{c}|z|} \int_{0}^{\infty} \frac{t \sqrt{c}}{\left(t^{2}+|z|^{2}\right)^{\frac{5}{4}}} d t \leq C_{2} \frac{e^{-\sigma|z|}}{|z|^{\frac{3}{2}}}
\end{aligned}
$$

where $\sigma>0$ is arbitrary, and the constants $C_{1}>0$ and $C_{2}>0$ are independent of $c>0$.

For $I_{B}(z)$, we observe that if $t>0$ satisfies

$$
c|z| \leq t \leq 2 \sqrt{\frac{m}{\lambda}} c^{\frac{3}{2}}|z|,
$$

then we have

$$
\sqrt{t^{2}+|z|^{2}} \geq \sqrt{1-\frac{\lambda}{m c^{2}}} t+\sqrt{\frac{\lambda}{m c^{2}}}|z| \geq\left(1-\frac{2 \lambda}{3 m c^{2}}\right) t+\sqrt{\frac{\lambda}{m c^{2}}}|z|
$$

for sufficiently large $c>0$. We thus obtain from (2.32) and (2.35) that for sufficiently large $c>0$,

$$
\begin{aligned}
I_{B}(z) & \leq \int_{c|z|}^{2 \sqrt{\frac{m}{\lambda} c^{\frac{3}{2}}|z|}} \frac{t \sqrt{c}}{\left(t^{2}+|z|^{2}\right)^{\frac{5}{4}}} e^{-\frac{\lambda}{3 c} t-\sqrt{\lambda m}|z|} d t \\
& \leq C_{3} e^{-\sqrt{\lambda m}|z|} \int_{c|z|}^{\infty} \frac{t \sqrt{c}}{\left(t^{2}+|z|^{2}\right)^{\frac{5}{4}}} d t \leq C_{4} \frac{e^{-\sqrt{\lambda m}|z|}}{\sqrt{|z|}},
\end{aligned}
$$

where the constants $C_{3}>0$ and $C_{4}>0$ are independent of $c>0$.

As for $I_{C}(z)$, we get that if $t>0$ satisfies

$$
C_{0} c^{\frac{1}{2}}|z|:=\frac{1}{4} \sqrt{\frac{m}{\lambda}} c^{\frac{1}{2}}|z| \leq t \leq c|z|,
$$

then we have

$$
\sqrt{t^{2}+|z|^{2}}=t \sqrt{1+\left(\frac{|z|}{t}\right)^{2}} \geq t+\left(\frac{1}{2}-\varepsilon\right) \frac{|z|^{2}}{t}
$$


for sufficiently large $c>0$, where $0<\varepsilon<\frac{1}{4}$ is arbitrary. We thus obtain from (2.32) and (2.37) that for sufficiently large $c>0$,

$$
\begin{aligned}
I_{C}(z) & \leq \sqrt{c} \int_{C_{0} c^{\frac{1}{2}}|z|}^{c|z|} \frac{t}{\left(t^{2}+|z|^{2}\right)^{\frac{5}{4}}} e^{-\frac{\lambda}{c} t-\left(\frac{1}{2}-\varepsilon\right) \frac{m c}{t}|z|^{2}} d t \\
& \leq-2 \sqrt{c} \int_{C_{0} c^{\frac{1}{2}}|z|}^{c|z|} e^{-\left(\frac{1}{2}-\varepsilon\right) \frac{m c}{t}|z|^{2}} d t^{-\frac{1}{2}} \\
& :=2 \sqrt{\frac{c}{|z|}} \int_{c^{-\frac{1}{2}}}^{C_{0}^{-\frac{1}{2}} c^{-\frac{1}{4}}} e^{-\left(\frac{1}{2}-\varepsilon\right) m c|z| s^{2}} d s .
\end{aligned}
$$

Note that if $\frac{1}{\sqrt{c}} \leq s$, then $2 \sqrt{c} d s \leq c d s^{2}$. We thus derive from (2.38) that for $\tau:=s^{2}>0$,

$$
I_{C}(z) \leq \frac{c}{\sqrt{|z|}} \int_{\frac{1}{c}}^{\frac{1}{c_{0} \sqrt{c}}} e^{-\left(\frac{1}{2}-\varepsilon\right) m c|z| \tau} d \tau \leq C_{5} \frac{e^{-\left(\frac{1}{2}-\varepsilon\right) m|z|}}{|z|^{\frac{3}{2}}},
$$

where the constant $C_{5}>0$ is also independent of $c>0$, and $0<\varepsilon<\frac{1}{4}$ is arbitrary as before.

Following (2.31), we now conclude from above that for $0<\delta_{0}:=\min \left\{\left(\frac{1}{2}-\varepsilon\right) m, \sqrt{\lambda m}\right\}$, where $0<\varepsilon<\frac{1}{4}$ is arbitrary, there exists a constant $M_{0}:=M_{0}\left(\delta_{0}\right)>0$ such that for all sufficiently large $c>0$,

$$
G_{c}(z) \leq M_{0} \frac{e^{-\delta_{0}|z|}}{\min \left\{|z|^{\frac{1}{2}},|z|^{\frac{3}{2}}\right\}}, \quad \text { if }|z| \geq \frac{1}{m c} .
$$

This further implies that for each $0<\delta_{1}<\min \left\{\frac{m}{2}, \sqrt{\lambda m}\right\}$, there exists a constant $M_{1}:=M_{1}\left(\delta_{1}\right)>0$ such that for $|z| \geq \frac{1}{m c}$,

$$
G_{c}(z) \leq M_{1} \frac{e^{-\delta_{1}|z|}}{|z|^{2}}
$$

uniformly for all sufficiently large $c>0$.

(2). Case of $|z| \leq \frac{1}{m c}$. In this case, we deduce from (2.22), (2.29) and (2.30) that for all sufficiently large $c>0$,

$$
\begin{aligned}
2 \pi^{2} G_{c}(z) \leq & \bar{M}_{2} m^{\frac{3}{2}} \sqrt{c} \int_{\frac{1}{m c}}^{\infty} \frac{t}{\left(t^{2}+|z|^{2}\right)^{\frac{5}{4}}} e^{-t\left(\frac{\lambda}{c}-m c\right)-m c \sqrt{t^{2}+|z|^{2}}} d t \\
& +\frac{\bar{M}_{1}}{c} \int_{0}^{\frac{1}{m c}} \frac{t}{\left(t^{2}+|z|^{2}\right)^{2}} e^{-\left(\frac{\lambda}{c}-m c\right) t} d t \\
:= & I_{1}(z)+I_{2}(z) .
\end{aligned}
$$

Similar to (2.31) and (2.40), one can obtain that for all sufficiently large $c>0$,

$$
I_{1}(z) \leq C_{6} \sqrt{c} \int_{0}^{\infty} \frac{t}{\left(t^{2}+|z|^{2}\right)^{\frac{5}{4}}} e^{-t\left(\frac{\lambda}{c}-m c\right)-m c \sqrt{t^{2}+|z|^{2}}} d t \leq \frac{M_{2}}{|z|^{2}}, \quad \text { if } \quad|z| \leq \frac{1}{m c},
$$


where the constants $C_{6}>0$ and $M_{2}>0$ are independent of $c>0$. As for $I_{2}(z)$, we infer that

$$
\begin{aligned}
I_{2}(z) & \leq \frac{C_{7}}{c} \int_{0}^{\frac{1}{m c}} \frac{t}{\left(t^{2}+|z|^{2}\right)^{2}} d t=\frac{C_{7}}{2 c|z|^{2}} \int_{0}^{\frac{1}{m c|z|}} \frac{1}{\left(s^{2}+1\right)^{2}} d s^{2} \\
& =\frac{C_{7}}{2 c|z|^{2}} \frac{(m c|z|)^{2}}{1+(m c|z|)^{2}} \leq \frac{C_{7}}{2 c|z|^{2}}, \quad \text { if } \quad|z| \leq \frac{1}{m c}
\end{aligned}
$$

where the constant $C_{7}>0$ is independent of $c>0$. We therefore derive from (2.42) and above that for all sufficiently large $c>0$,

$$
G_{c}(z) \leq \frac{M_{3}}{|z|^{2}}, \text { if }|z| \leq \frac{1}{m c},
$$

where the constant $M_{3}>0$ is also independent of $c>0$.

We finally conclude from (2.41) and (2.44) that (2.25) holds true, and we are done.

Proof of Lemma 2.1. We first prove that the positive solution $Q_{c}>0$ of (2.6), where the Lagrange multiplier $\mu_{c}$ is as in (2.7), satisfies the following exponential decay

$$
\left|Q_{c}\right| \leq C e^{-\delta|x|} \text { in } \mathbb{R}^{3}
$$

uniformly for all sufficiently large $c>0$, where the constants $\delta>0$ and $C=C(\delta)>0$ are independent of $c>0$. Actually, recall from (2.6) that $Q_{c}$ can be rewritten as

$$
Q_{c}(x)=-\int_{\mathbb{R}^{3}} G_{c}(x-y) V(y) Q_{c}(y) d y,
$$

where $G_{c}(x-y)$ is the Green's function of $\left(\bar{H}_{c}+\left(-\mu_{c}\right)\right)^{-1}$ defined by (2.23) , and the potential $V(x):=-\left(\frac{1}{|x|} * Q_{c}^{2}\right)(x)$ satisfies $V(x) \in C^{0}\left(\mathbb{R}^{3}\right)$ and $\lim _{|x| \rightarrow \infty} V(x)=0$. Since $-\mu_{c} \rightarrow \lambda>0$ as $c \rightarrow \infty$ in view of (2.7), $G_{c}(x-y)$ satisfies the exponential decay of Lemma 2.3. Following the above properties, the uniformly exponential decay (2.45) as $c \rightarrow \infty$ can be proved in a similar way of [14, Appendix C] and [24, Theorem 2.1], where the Slaggie-Wichmann method (e.g. [24]) is employed.

To finish the proof of Lemma 2.1, we next rewrite the solution $\varphi_{c} \in H^{\frac{1}{2}}\left(\mathbb{R}^{3}\right)$ of (2.9) as

$$
\varphi_{c}(x)=\left(\bar{H}_{c}+\left(-\mu_{c}\right)\right)^{-1}\left(V_{1} \varphi_{c}+2 k_{1} V_{2}\left(\varphi_{c}\right)+k_{2}(c) Q_{c}\right),
$$

where the operator $\bar{H}_{c}$ satisfies (2.23) as before and

$$
V_{1}=\frac{1}{|x|} * Q_{c}^{2}, \quad V_{2}\left(\varphi_{c}\right)=\left\{\frac{1}{|x|} *\left(Q_{c} \varphi_{c}\right)\right\} Q_{c} .
$$

Here $\mu_{c} \in \mathbb{R}$ satisfies (2.7), $k_{1} \geq 0$, and $k_{2}(c) \in \mathbb{R}$ is bounded uniformly in $c>0$. Note from (2.47) that $\varphi_{c} \in H^{\frac{1}{2}}\left(\mathbb{R}^{3}\right)$ solves

$$
\varphi_{c}(x)=\int_{\mathbb{R}^{3}} G_{c}(x-y)\left(V_{1} \varphi_{c}+k_{1} V_{2}\left(\varphi_{c}\right)+k_{2}(c) Q_{c}\right)(y) d y,
$$


where the Green's function $G_{c}(\cdot)$ of $\left(\bar{H}_{c}+\left(-\mu_{c}\right)\right)^{-1}$ satisfies as before the uniformly exponential decay of Lemma 2.3 in view of (2.7). Since $Q_{c}$ satisfies the uniformly exponential decay (2.45) as $c \rightarrow \infty$, the uniformly exponential decay (2.10) of $\varphi_{c}$ as $c \rightarrow \infty$ can be further proved in a similar way of [13, Lemma 4.9]. We omit the detailed proof for simplicity. This completes the proof of Lemma 2.1.

\section{Proof of Theorem 1.2}

Following the refined estimates of previous section, in this section we shall complete the proof of Theorem 1.2. We begin with the following two lemmas.

Lemma 3.1. Suppose $Q_{\infty}$ is the unique radially symmetric positive solution of (2.3) and let the operator $L_{+}$be defined by 2.4). Then we have

$$
L_{+}\left(x \cdot \nabla Q_{\infty}+2 Q_{\infty}\right)=-2 \lambda Q_{\infty},
$$

where $\lambda>0$ is as in (2.3).

Proof. Direct calculations give that

$$
\begin{aligned}
-\Delta\left(x \cdot \nabla Q_{\infty}\right) & =-\sum_{i=1}^{3} \partial_{i}\left(\partial_{i}\left(\sum_{j=1}^{3} x_{j} \partial_{j} Q_{\infty}\right)\right)=-\sum_{i, j=1}^{3} \partial_{i}\left(\delta_{i j} \partial_{j} Q_{\infty}+x_{j} \partial_{i j} Q_{\infty}\right) \\
& =-\sum_{i, j=1}^{3}\left(2 \delta_{i j} \partial_{i j} Q_{\infty}+x_{j} \partial_{i i j} Q_{\infty}\right)=-2 \Delta Q_{\infty}-x \cdot \nabla\left(\Delta Q_{\infty}\right),
\end{aligned}
$$

and

$$
2 Q_{\infty}\left[|x|^{-1} *\left(Q_{\infty} x \cdot \nabla Q_{\infty}\right)\right]=Q_{\infty}\left[|x|^{-1} *\left(x \cdot \nabla Q_{\infty}^{2}\right)\right] .
$$

We then have

$$
\begin{aligned}
L_{+}\left(x \cdot \nabla Q_{\infty}\right) & =-\frac{1}{m} \Delta Q_{\infty}-\frac{1}{2 m} x \cdot \nabla\left(\Delta Q_{\infty}\right)+\lambda x \cdot \nabla Q_{\infty} \\
& -\left(|x|^{-1} * Q_{\infty}^{2}\right)\left(x \cdot \nabla Q_{\infty}\right)-Q_{\infty}\left[|x|^{-1} *\left(x \cdot \nabla Q_{\infty}^{2}\right)\right] .
\end{aligned}
$$

Taking the action $x \cdot \nabla$ on (2.3), we deduce that

$$
-\frac{1}{2 m} x \cdot \nabla\left(\Delta Q_{\infty}\right)+\lambda x \cdot \nabla Q_{\infty}=\left(|x|^{-1} * Q_{\infty}^{2}\right)\left(x \cdot \nabla Q_{\infty}\right)+Q_{\infty} x \cdot\left(|x|^{-1} * \nabla Q_{\infty}^{2}\right) .
$$

Together with (3.2), this indicates that

$$
L_{+}\left(x \cdot \nabla Q_{\infty}\right)=-\frac{1}{m} \Delta Q_{\infty}+Q_{\infty} x \cdot\left(|x|^{-1} * \nabla Q_{\infty}^{2}\right)-Q_{\infty}\left[|x|^{-1} *\left(x \cdot \nabla Q_{\infty}^{2}\right)\right] .
$$


Since

$$
\begin{aligned}
& x \cdot\left(|x|^{-1} * \nabla Q_{\infty}^{2}\right)-|x|^{-1} *\left(x \cdot \nabla Q_{\infty}^{2}\right) \\
= & \int_{\mathbb{R}^{3}} \frac{x \cdot \nabla Q_{\infty}^{2}(y)}{|x-y|} d y-\int_{\mathbb{R}^{3}} \frac{y \cdot \nabla Q_{\infty}^{2}(y)}{|x-y|} d y \\
= & \int_{\mathbb{R}^{3}} \frac{(x-y) \cdot \nabla Q_{\infty}^{2}(y)}{|x-y|} d y=-\sum_{i=1}^{3} \int_{\mathbb{R}^{3}} \partial_{y_{i}}\left(\frac{x-y}{|x-y|}\right) Q_{\infty}^{2}(y) d y \\
= & 2 \int_{\mathbb{R}^{3}} \frac{Q_{\infty}^{2}(y)}{|x-y|} d y=2|x|^{-1} * Q_{\infty}^{2},
\end{aligned}
$$

it follows from (3.3) that

$$
L_{+}\left(x \cdot \nabla Q_{\infty}\right)=-\frac{1}{m} \Delta Q_{\infty}+2\left(|x|^{-1} * Q_{\infty}^{2}\right) Q_{\infty} .
$$

Moreover, recall from (2.3) that

$$
L_{+} Q_{\infty}=\left(-\frac{1}{2 m} \Delta+\lambda-3|x|^{-1} *\left|Q_{\infty}\right|^{2}\right) Q_{\infty}=-2\left(|x|^{-1} * Q_{\infty}^{2}\right) Q_{\infty} .
$$

Combining (3.5) with (3.6) thus yields that

$$
L_{+}\left(x \cdot \nabla Q_{\infty}+2 Q_{\infty}\right)=-\frac{1}{m} \Delta Q_{\infty}-2\left(|x|^{-1} * Q_{\infty}^{2}\right) Q_{\infty}=-2 \lambda Q_{\infty},
$$

and the proof of this lemma is therefore complete.

Lemma 3.2. Let $Q_{c}$ be a radially symmetric positive minimizer of $\bar{e}(c)$ defined in (1.9)). Then we have the following Pohozaev identity

$$
-m^{2} c^{4}\left\langle\left(-c^{2} \Delta+m^{2} c^{4}\right)^{-\frac{1}{2}} Q_{c}, Q_{c}\right\rangle+m c^{2} \int_{\mathbb{R}^{3}}\left|Q_{c}\right|^{2} d x+\bar{e}(c)=0 .
$$

Proof. In the proof of this lemma, we denote $Q_{c}$ by $Q$ for convenience. We first note that

$$
x \cdot \nabla Q(x)=\left.\frac{d}{d \lambda} Q_{\lambda}(x)\right|_{\lambda=1}, \quad \text { where } Q_{\lambda}(x):=Q(\lambda x) .
$$

Multiplying $x \cdot \nabla Q(x)$ on both sides of (2.6) and integrating over $\mathbb{R}^{3}$, we have

$$
\begin{aligned}
& \left\langle\sqrt{-c^{2} \Delta+m^{2} c^{4}} Q, x \cdot \nabla Q\right\rangle \\
= & \left.\frac{d}{d \lambda}\left\langle\sqrt{-c^{2} \Delta+m^{2} c^{4}} Q, Q \lambda\right\rangle\right|_{\lambda=1} \\
= & \left.\frac{d}{d \lambda}\left\langle\left(-c^{2} \Delta+m^{2} c^{4}\right)^{\frac{1}{4}} Q,\left(-c^{2} \Delta+m^{2} c^{4}\right)^{\frac{1}{4}} Q_{\lambda}\right\rangle\right|_{\lambda=1} \\
\sqrt{\lambda}=x^{\prime} & \left.\frac{d}{d \lambda} \lambda^{-1}\left\langle\left(-c^{2} \Delta+\frac{m^{2} c^{4}}{\lambda}\right)^{\frac{1}{4}} Q\left(\frac{x}{\sqrt{\lambda}}\right),\left(-c^{2} \Delta+\frac{m^{2} c^{4}}{\lambda}\right)^{\frac{1}{4}} Q(\sqrt{\lambda} x)\right\rangle\right|_{\lambda=1} \\
= & -\int_{\mathbb{R}^{3}}\left|\left(-c^{2} \Delta+m^{2} c^{4}\right)^{\frac{1}{4}} Q\right|^{2} d x \\
& -\frac{m^{2} c^{4}}{2}\left\langle\left(-c^{2} \Delta+m^{2} c^{4}\right)^{-\frac{3}{4}} Q,\left(-c^{2} \Delta+m^{2} c^{4}\right)^{\frac{1}{4}} Q\right\rangle \\
= & -\left\langle\sqrt{-c^{2} \Delta+m^{2} c^{4}} Q, Q\right\rangle-\frac{m^{2} c^{4}}{2}\left\langle\left(-c^{2} \Delta+m^{2} c^{4}\right)^{-\frac{1}{2}} Q, Q\right\rangle .
\end{aligned}
$$


Moreover, we derive from the exponential decay (2.11) that

$$
\begin{aligned}
\int_{\mathbb{R}^{3}}\left(|x|^{-1} * Q^{2}\right) Q(x \cdot \nabla Q) d x & =\frac{1}{2} \int_{\mathbb{R}^{3}}\left(|x|^{-1} * Q^{2}\right)\left(x \cdot \nabla Q^{2}\right) d x \\
=-\frac{3}{2} \int_{\mathbb{R}^{3}}\left(|x|^{-1} * Q^{2}\right) Q^{2} d x & -\frac{1}{2} \int_{\mathbb{R}^{3}}\left[\left(|x|^{-1} * \nabla Q^{2}\right) \cdot x\right] Q^{2} d x \\
=-\frac{3}{2} \int_{\mathbb{R}^{3}}\left(|x|^{-1} * Q^{2}\right) Q^{2} d x & -\frac{1}{2}\left[2 \int_{\mathbb{R}^{3}}\left(|x|^{-1} * Q^{2}\right) Q^{2} d x\right. \\
& \left.+\iint_{\mathbb{R}^{3}} \frac{y \cdot \nabla Q^{2}(y)}{|x-y|} Q^{2}(x) d y d x\right],
\end{aligned}
$$

where the argument of deriving (3.4) is used in the last equality. Since

$$
\iint_{\mathbb{R}^{3}} \frac{y \cdot \nabla Q^{2}(y)}{|x-y|} Q^{2}(x) d y d x=\int_{\mathbb{R}^{3}}\left(|x|^{-1} * Q^{2}\right)\left(x \cdot \nabla Q^{2}\right) d x,
$$

we obtain from (3.9) that

$$
\int_{\mathbb{R}^{3}}\left(|x|^{-1} * Q^{2}\right) Q(x \cdot \nabla Q) d x=-\frac{5}{4} \int_{\mathbb{R}^{3}}\left(|x|^{-1} * Q^{2}\right) Q^{2} d x .
$$

One can easily check that

$$
\int_{\mathbb{R}^{3}} Q(x \cdot \nabla Q) d x=-\frac{3}{2} \int_{\mathbb{R}^{3}} Q^{2} d x .
$$

Thus, it follows from (2.6), (3.8) and (3.10) that

$$
\begin{aligned}
& -\left\langle\sqrt{-c^{2} \Delta+m^{2} c^{4}} Q, Q\right\rangle-\frac{m^{2} c^{4}}{2}\left\langle\left(-c^{2} \Delta+m^{2} c^{4}\right)^{-\frac{1}{2}} Q, Q\right\rangle \\
& =-\frac{3}{2}\left(m c^{2}+\mu_{c}\right) \int_{\mathbb{R}^{3}} Q^{2} d x-\frac{5}{4} \int_{\mathbb{R}^{3}}\left(|x|^{-1} * Q^{2}\right) Q^{2} d x .
\end{aligned}
$$

By (3.11), multiplying $Q$ on both sides of (2.6) and integrating over $\mathbb{R}^{3}$ yield that

$$
\begin{aligned}
& -m^{2} c^{4}\left\langle\left(-c^{2} \Delta+m^{2} c^{4}\right)^{-\frac{1}{2}} Q, Q\right\rangle \\
& +\left(m c^{2}+\mu_{c}\right) \int_{\mathbb{R}^{3}}|Q|^{2} d x+\frac{1}{2} \int_{\mathbb{R}^{3}}\left(|x|^{-1} * Q^{2}\right) Q^{2} d x=0 .
\end{aligned}
$$

We also derive from (2.6) that

$$
\begin{aligned}
\mu_{c} \int_{\mathbb{R}^{3}}|Q|^{2} d x & =\left\langle\sqrt{-c^{2} \Delta+m^{2} c^{4}} Q, Q\right\rangle-m c^{2} \int_{\mathbb{R}^{3}}|Q|^{2} d x-\int_{\mathbb{R}^{3}}\left(|x|^{-1} * Q^{2}\right) Q^{2} d x \\
& =\bar{e}(c)-\frac{1}{2} \int_{\mathbb{R}^{3}}\left(|x|^{-1} * Q^{2}\right) Q^{2} d x,
\end{aligned}
$$

which therefore implies that (3.7) holds true by applying (3.12).

Following previous estimates, we are now ready to finish the proof of Theorem 1.2 . 
Proof of Theorem 1.2. Up to the phase and translation, it suffices to prove that $\bar{e}(c)$ in (1.9) admits a unique positive minimizer for sufficiently large $c>0$. On the contrary, suppose that $Q_{1 c}$ and $Q_{2 c}$ are two different radially symmetric (about the origin) positive minimizers of problem (1.9), where $m>0$ is fixed.

Then $Q_{i c} \in H^{s}\left(\mathbb{R}^{3}\right)$, where $s \geq \frac{1}{2}$, satisfies the following equation

$$
\left(\sqrt{-c^{2} \Delta+m^{2} c^{4}}-m c^{2}\right) Q_{i c}-\left(|x|^{-1} * Q_{i c}^{2}\right) Q_{i c}=\mu_{i c} Q_{i c} \text { in } \mathbb{R}^{3}, i=1,2,
$$

where $\mu_{i c} \in \mathbb{R}$ is the Lagrange multiplier associated to $Q_{i c}$ for $i=1,2$. Since $Q_{1 c} \not \equiv Q_{2 c}$ in $\mathbb{R}^{3}$, we define

$$
w_{c}(x):=\frac{Q_{1 c}(x)-Q_{2 c}(x)}{\left\|Q_{1 c}-Q_{2 c}\right\|_{L^{\infty}\left(\mathbb{R}^{3}\right)}} \text { in } \mathbb{R}^{3} .
$$

It then follows from (3.13) that

$$
\begin{aligned}
& \left(\sqrt{-c^{2} \Delta+m^{2} c^{4}}-m c^{2}\right) w_{c}-\left(|x|^{-1} * Q_{1 c}^{2}\right) w_{c}-\left\{|x|^{-1} *\left[\left(Q_{1 c}+Q_{2 c}\right) w_{c}\right]\right\} Q_{2 c} \\
& =\mu_{2 c} w_{c}+\frac{\mu_{1 c}-\mu_{2 c}}{\left\|Q_{1 c}-Q_{2 c}\right\|_{L^{\infty}\left(\mathbb{R}^{3}\right)}} Q_{1 c} \text { in } \mathbb{R}^{3} .
\end{aligned}
$$

Recall from (2.7) that

$$
\lim _{c \rightarrow \infty} \mu_{i c}=-\lambda<0, i=1,2 .
$$

We also note from (3.13) that

$$
\mu_{i c}=\bar{e}(c)-\frac{1}{2} \int_{\mathbb{R}^{3}}\left(|x|^{-1} * Q_{i c}^{2}\right) Q_{i c}^{2} d x, i=1,2,
$$

which implies that

$$
\begin{aligned}
\frac{\mu_{1 c}-\mu_{2 c}}{\left\|Q_{1 c}-Q_{2 c}\right\|_{L^{\infty}\left(\mathbb{R}^{3}\right)}}=-\frac{1}{2} \int_{\mathbb{R}^{3}} & \left\{\left(|x|^{-1} * Q_{1 c}^{2}\right)\left(Q_{1 c}+Q_{2 c}\right) w_{c}\right. \\
\left.+\left[|x|^{-1} *\left(\left(Q_{1 c}+Q_{2 c}\right) w_{c}\right)\right] Q_{2 c}^{2}\right\} d x:=k_{2}(c) \in \mathbb{R} &
\end{aligned}
$$

where $k_{2}(c)$ is bounded uniformly in $c>0$ by (2.11) and (2.16). Applying Lemma 2.1 to the equation (3.15), we then deduce from (3.16) and (3.17) that there exist $\delta>0$ and $C=C(\delta)>0$, which are independent of $c>0$, such that

$$
\left|w_{c}\right| \leq C e^{-\delta|x|} \text { in } \mathbb{R}^{3}
$$

uniformly as $c \rightarrow \infty$. Similar to the proof of Lemma 2.1, following (3.15) to consider the equation of $\frac{\partial w_{c}}{\partial x_{i}}(i=1,2,3)$, we further derive from (3.16)-(3.18) that there exist $\delta_{1}>0$ and $C_{1}=C_{1}\left(\delta_{1}\right)>0$, which are independent of $c>0$, such that

$$
\left|\nabla w_{c}\right| \leq C_{1} e^{-\delta_{1}|x|} \text { in } \mathbb{R}^{3}
$$

uniformly as $c \rightarrow \infty$. 
Rewrite the equation (3.15) as

$$
\begin{aligned}
\left(\bar{H}_{c}+\mu\right) w_{c}= & \left\{\mu+\mu_{2 c}+\left(|x|^{-1} * Q_{1 c}^{2}\right)\right\} w_{c} \\
& +k_{2}(c) Q_{1 c}+\left\{|x|^{-1} *\left[\left(Q_{1 c}+Q_{2 c}\right) w_{c}\right]\right\} Q_{2 c} \text { in } \mathbb{R}^{3}
\end{aligned}
$$

for any $\mu \in \mathbb{R}$, where the uniformly bounded function $k_{2}(c) \in \mathbb{R}$ is as in (3.17), and the operator $\bar{H}_{c}$ satisfies

$$
\bar{H}_{c}:=\sqrt{-c^{2} \Delta+m^{2} c^{4}}-m c^{2} .
$$

Since $\left\|w_{c}\right\|_{L^{\infty}\left(\mathbb{R}^{3}\right)} \leq 1$ for all $c>0$, we obtain from (2.11), (2.16) and (2.18) that for sufficiently large $c>0$,

$$
\left\||x|^{-1} * Q_{1 c}^{2}\right\|_{L^{\infty}\left(\mathbb{R}^{3}\right)}<M, \quad\left\||x|^{-1} *\left[\left(Q_{1 c}+Q_{2 c}\right) w_{c}\right]\right\|_{L^{\infty}\left(\mathbb{R}^{3}\right)}<M,
$$

where $M>0$ is independent of $c>0$. In view of (2.16) and (3.21), the similar argument of [14, Theorem 4.1(i)] or [7, Proposition 4.2] applied to (3.20) and (3.16) yields that

$$
w_{c} \in H^{s}\left(\mathbb{R}^{3}\right) \text { for all } s \geq \frac{1}{2},
$$

which further implies the uniform smoothness of $w_{c}$ in $c>0$.

We next rewrite the equation (3.15) as

$$
\begin{aligned}
& \left(-\frac{1}{2 m} \Delta-\mu_{2 c}\right) w_{c}-\left(|x|^{-1} * Q_{1 c}^{2}\right) w_{c}-\left\{|x|^{-1} *\left[\left(Q_{1 c}+Q_{2 c}\right) w_{c}\right]\right\} Q_{2 c} \\
& =k_{2}(c) Q_{1 c}-F_{c}(\nabla) w_{c} \text { in } \mathbb{R}^{3},
\end{aligned}
$$

where the uniformly bounded function $k_{2}(c) \in \mathbb{R}$ is again as in (3.17), and the pseudodifferential operator $F_{c}(\nabla)$ is the same as (2.15) with the symbol

$$
F_{c}(\xi):=\sqrt{c^{2}|\xi|^{2}+m^{2} c^{4}}-m c^{2}-\frac{|\xi|^{2}}{2 m}, \quad \xi \in \mathbb{R}^{3} .
$$

Note that $F_{c}(\nabla)$ satisfies the estimate (2.17). We then derive from (2.17) and (3.22) that

$$
\left\|F_{c}(\nabla) w_{c}\right\|_{H^{s}\left(\mathbb{R}^{3}\right)} \leq \frac{M_{1}}{m^{3} c^{2}}\left\|w_{c}\right\|_{H^{s+4}\left(\mathbb{R}^{3}\right)}<\frac{M_{2}}{m^{3} c^{2}} \text { for all } s \geq \frac{1}{2},
$$

where $M_{1}>0$ and $M_{2}>0$ are independent of $c>0$.

Using the uniformly exponential decays (2.11) and (3.18), by the standard elliptic regularity we derive from (2.16), (3.23) and (3.24) that $\left\|w_{c}\right\|_{C_{\text {loc }}^{\alpha}\left(\mathbb{R}^{3}\right)} \leq M_{3}$ for some $\alpha \in(0,1)$, where the constant $M_{3}>0$ is independent of $c$. Therefore, there exists a function $w_{0}=w_{0}(x)$ such that up to a subsequence if necessary, we have

$$
w_{c} \rightarrow w_{0} \text { in } C_{\mathrm{loc}}\left(\mathbb{R}^{3}\right) \text { as } c \rightarrow \infty \text {. }
$$

Moreover, applying the estimates (3.16), (3.17) and (3.24), we deduce from (3.23) that $w_{0}$ satisfies

$$
L_{+} w_{0}=-Q_{\infty} \int_{\mathbb{R}^{3}}\left\{\left(|x|^{-1} * Q_{\infty}^{2}\right) Q_{\infty} w_{0}+\left[|x|^{-1} *\left(Q_{\infty} w_{0}\right)\right] Q_{\infty}^{2}\right\} d x
$$


where the uniformly exponential decay (2.11) is also used. Applying (2.5) and Lemma 3.1 , we now derive from (3.25) that there exist constants $b_{0}$ and $c_{i}(i=1,2,3)$ such that

$$
w_{0}=b_{0}\left(x \cdot \nabla Q_{\infty}+2 Q_{\infty}\right)+\sum_{i=1}^{3} c_{i} \frac{\partial Q_{\infty}}{\partial x_{i}} .
$$

Further, since $Q_{1 c}$ and $Q_{2 c}$ are both radially symmetric in $|x|$ for all $c>0$, the definition of $w_{c}$ implies that $w_{0}$ is also radially symmetric in $|x|$, i.e., $w_{0} \in L_{\text {rad }}^{2}\left(\mathbb{R}^{3}\right)$. Applying 26 , Proposition 2], it then follows from the above expression that

$$
w_{0}=b_{0}\left(x \cdot \nabla Q_{\infty}+2 Q_{\infty}\right) \text { for some } b_{0} \in \mathbb{R} .
$$

We next prove $b_{0}=0$ in (3.26) so that $w_{0} \equiv 0$ in $\mathbb{R}^{3}$. Indeed, multiplying (3.7) by $Q_{1 c}$ and $Q_{2 c}$ respectively, and integrating over $\mathbb{R}^{3}$, we obtain that

$$
\begin{aligned}
&-m c^{2}\left\langle\left(-c^{2} \Delta+m^{2} c^{4}\right)^{-\frac{1}{2}} w_{c}, Q_{1 c}\right\rangle-m c^{2}\left\langle\left(-c^{2} \Delta+m^{2} c^{4}\right)^{-\frac{1}{2}} Q_{2 c}, w_{c}\right\rangle \\
&+\int_{\mathbb{R}^{3}} w_{c}\left(Q_{1 c}+Q_{2 c}\right) d x=0 .
\end{aligned}
$$

Moreover, since $\frac{1}{\sqrt{1+t}}=1-\frac{t}{2}+O\left(t^{2}\right)$ as $t \rightarrow 0$ and $w_{c}$ is smooth for all $c>0$, we have

$$
m c^{2}\left(-c^{2} \Delta+m^{2} c^{4}\right)^{-\frac{1}{2}}=\left(1-\frac{\Delta}{m^{2} c^{2}}\right)^{-\frac{1}{2}}=\left[1+\frac{\Delta}{2 m^{2} c^{2}}+O\left(\frac{1}{m^{4} c^{4}}\right)\right] \text { as } c \rightarrow \infty .
$$

Putting it into (3.27) yields that

$$
-\frac{1}{2 m} \int_{\mathbb{R}^{3}}\left(Q_{1 c} \Delta w_{c}+w_{c} \Delta Q_{2 c}\right) d x+O\left(\frac{1}{m^{3} c^{2}}\right)=0 \text { as } c \rightarrow \infty,
$$

where the exponential decays (2.11), (3.18) and (3.19) are used again. Applying (2.11), (2.12) and (3.22), it then follows from above that

$$
\int_{\mathbb{R}^{3}} \nabla w_{0} \nabla Q_{\infty} d x=0
$$

We thus conclude from (3.26) and (3.28) that

$$
0=b_{0} \int_{\mathbb{R}^{3}} \nabla\left(x \cdot \nabla Q_{\infty}+2 Q_{\infty}\right) \nabla Q_{\infty} d x=\frac{3}{2} b_{0} \int_{\mathbb{R}^{3}}\left|\nabla Q_{\infty}\right|^{2} d x
$$

which therefore implies that $b_{0}=0$ in $(3.26)$ and thus $w_{0} \equiv 0$ in $\mathbb{R}^{3}$.

We are now ready to derive a contradiction. In fact, let $y_{c} \in \mathbb{R}^{3}$ be a point satisfying $\left|w_{c}\left(y_{c}\right)\right|=\left\|w_{c}\right\|_{L^{\infty}\left(\mathbb{R}^{3}\right)}=1$. Since it follows from (3.18) that $w_{c}$ admits the exponential decay uniformly for all $c>0$, we have $\left|y_{c}\right| \leq M$ uniformly in $c$ for some constant $M>0$. Therefore, we obtain that $w_{c} \rightarrow w_{0} \not \equiv 0$ uniformly on $\mathbb{R}^{3}$ as $c \rightarrow \infty$, which however contradicts to the fact that $w_{0} \equiv 0$ on $\mathbb{R}^{3}$. This completes the proof of Theorem 1.2 .

Acknowledgements: The authors thank Professor Enno Lenzmann very much for his helpful discussions on the subject of the present work. 


\section{References}

[1] M. Abramowitz and I. A. Stegun, Handbook of mathematical functions with formulas, graphs, and mathematical tables, New York: Dover Publications Inc., 1992, Reprint of the 1972 edition.

[2] W. H. Aschbacher, J. Fröhlich, G. M. Graf, K. Schnee and M. Troyer, Symmetry breaking regime in the nonlinear Hartree equation, J. Math. Phys. 43 (2002), 3879.

[3] X. Cabré and Y. Sire, Nonlinear equations for fractional Laplacians, I: Regularity, maximum principles, and Hamiltonian estimates, Ann. Inst. H. Poincaré Anal. Non Linéaire 31 (2014), 23-53.

[4] L. Caffarellia and L. Silvestre, An extension problem related to the fractional Laplacian, Comm. Partial Differential Equations 32 (2007), 1245-1260.

[5] D. M. Cao and Y. M. Su, Minimal blow-up solutions of mass-critical inhomogeneous Hartree equation, J. Math. Phys. 54 (2013), 121511.

[6] R. Carles, W. Lucha and E. Moulay, Higher-order Schrödinger and Hartree-Fock equations, J. Math. Phys. 56 (2015), 122301.

[7] W. Choi, Y. Hong and J. Seok, Optimal convergence rate and regularity of nonrelativistic limit for the nonlinear pseudo-relativistic equations, J. Funct. Anal. 274 (2018), no. 3, 695-722.

[8] V. Coti-Zelati and M. Nolasco, Existence of ground states for nonlinear, pseudorelativistic Schrödinger equations, Rend. Lincei Mat. Appl. 22 (2011), 51-72.

[9] Y. B. Deng, C. S. Lin and S. Yan, On the prescribed scalar curvature problem in $\mathbb{R}^{N}$, local uniqueness and periodicity, J. Math. Pures Appl. 104 (2015), no. 6, 1013-1044.

[10] A. Elgart and B. Schlein, Mean field dynamics of boson stars, Comm. Pure Appl. Math. 60 (2007), no. 4, 500-545.

[11] R. Frank and E. Lenzmann, Uniqueness and nondegeneracy of ground states for $(-\Delta)^{s} Q+Q-Q^{\alpha+1}=0$ in $\mathbb{R}$, Acta Math. 210 (2013), no. 2, 261-318.

[12] R. Frank, E. Lenzmann and L. Silvestre, Uniqueness of radial solutions for the fractional Laplacian, Comm. Pure Appl. Math. 69 (2016), no. 9, 1671-1726.

[13] J. Fröhlich, B. L. G. Jonsson and E. Lenzmann, Effective dynamics for boson stars, Nonlinearity 20 (2007), no. 5, 1031-1075.

[14] J. Fröhlich, B. L. G. Jonsson and E. Lenzmann, Boson stars as solitary waves, Comm. Math. Phys. 274 (2007), no. 1, 1-30.

[15] J. Fröhlich and E. Lenzmann, Blowup for nonlinear wave equations describing boson stars, Comm. Pure Appl. Math. 60 (2007), no. 11, 1691-1705. 
[16] J. Fröhlich, T.-P. Tsai and H.-T. Yau, On the point-particle (Newtonian) limit of the non-linear Hartree equation, Comm. Math. Phys. 225 (2002), no. 2, 223-274.

[17] D. Gilbarg and N. S. Trudinger, Elliptic Partial Differential Equations of Second Order, Springer, (1997).

[18] M. Grossi, On the number of single-peak solutions of the nonlinear Schrödinger equations, Ann. Inst H. Poincar Anal. Non Linaire 19 (2002), 261-280.

[19] Y. J. Guo, C. S. Lin and J. C. Wei, Local uniqueness and refined spike profiles of ground states for two-dimensional attractive Bose-Einstein condensates, SIAM J. Math. Anal. 49 (2017), no. 5, 3671-3715.

[20] Y. J. Guo and R. Seiringer, On the mass concentration for Bose-Einstein condensates with attractive interactions, Lett. Math. Phys. 104 (2014), 141-156.

[21] Y. J. Guo, Z. Q. Wang, X. Y. Zeng and H. S. Zhou, Properties for ground states of attractive Gross-Pitaevskii equations with multi-well potentials, Nonlinearity $\mathbf{3 1}$ (2018), 957-979.

[22] Y. J. Guo, X. Y. Zeng and H. S. Zhou, Energy estimates and symmetry breaking in attractive Bose-Einstein condensates with ring-shaped potentials, Ann. Inst. H. Poincaré Anal. Non Linéaire 33 (2016), no. 3, 809-828.

[23] Y. J. Guo and X. Y. Zeng, Ground states of pseudo-relativistic boson stars under the critical stellar mass, Ann. Inst. H. Poincar Anal. Non Linaire 34 (2017), no. 6, 1611-1632.

[24] P. D. Hislop, Exponential decay of two-body eigenfunctions: a review, Proceedings of the Symposium on Mathematical Physics and Quantum Field Theory (Berkeley, CA, 1999) (San Marcos, TX), Electron. J. Differ. Equ. Conf. Vol. 4, Southwest Texas State Univ. (2000), 265-288.

[25] E. Lenzmann, Well-posedness for semi-relativistic Hartree equations of critical type, Math. Phys. Anal. Geom. 10 (2007), no. 1, 43-64.

[26] E. Lenzmann, Uniqueness of ground states for pseudorelativistic Hartree equations, Anal. PDE 2 (2009), no. 1, 1-27.

[27] E. H. Lieb, Existence and uniqueness of the minimizing solution of Choquard's nonlinear equation, Studies in Appl. Math. 57 (1976/77), no. 2, 93-105.

[28] E. H. Lieb and M. Loss, Analysis, Graduate Studies in Mathematics, Vol. 14, American Mathematical Society, Providence, RI, 2001.

[29] E. H. Lieb and H.-T. Yau, The Chandrasekhar theory of stellar collapse as the limit of quantum mechanics, Comm. Math. Phys. 112 (1987), no. 1, 147-174.

[30] E. H. Lieb and H.-T. Yau, A rigorous examination of the Chandrasekhar theory of stellar collapse, Astrophysical J. 323 (1987), no. 1, 140-144. 
[31] M. Maeda, On the symmetry of the ground states of nonlinear Schrödinger equation with potential, Adv. Nonlinear Stud. 10 (2010), 895-925.

[32] V. Moroz and J. V. Schaftingen, Ground states of nonlinear Choquard equations: Existence, qualitative properties and decay asymptotics, J. Funct. Anal. 265 (2013), no. 1, 153-184.

[33] D. T. Nguyen, On blow-up profile of ground states of boson stars with external potential, J. Stat. Phys. 169 (2017), no. 2, 395-422.

[34] P. L. Lions, The concentration-compactness principle in the calculus of variations. The locally compact case, Part I: Ann. Inst. H. Poincaré Anal. Non Linéaire 1 (1984), 109-145. Part II: Ann. Inst. H. Poincaré Anal. Non Linéaire 1 (1984), 223-283.

[35] E. L. Slaggie and E. H. Wichmann, Asymptotic properties of the wave function for a bound nonrelativistic three-body system J. Math. Phys. 3 (1962), 946-968.

[36] M. Struwe, Variational Methods: Applications to Nonlinear Partial Differential Equations and Hamiltonian Systems, Ergebnisse Math. 34, Springer (2008).

[37] J. F. Yang and J. G. Yang, Existence and mass concentration of pseudo-relativistic Hartree equation, J. Math. Phys. 58 (2017), no. 8, 081501. 